\title{
Prevalence and Determinants of Acute Respiratory Infections among Children under Five Years in Rural Settings of The Gambia: Evidence from a National Survey
}

\author{
Amadou Barrow ${ }^{1,2,3}{ }^{*}{ }^{\circ}$, Afape Ayobami ${ }^{4}$, Precious Chidozie Azubuike ${ }^{5}$, Dalanda Cham ${ }^{1,3}$

\footnotetext{
${ }^{1}$ Department of Public \& Environmental Health, School of Medicine \& Allied Health Sciences, University of The Gambia, Kanifing, The Gambia

2 School of Public Health, Gambia College, Brikama campus, West Coast Region, The Gambia

${ }^{3}$ Epidemiology \& Disease Control Unit, Ministry of Health, Kotu, The Gambia

${ }^{4}$ Kaduna State AIDS Control Agency, Ministry of Health and Human Services, Kaduna State, Nigeria

${ }^{5}$ Department of Reproductive Health, Institute of Life and Earth Sciences, Pan African University, Ibadan, Nigeria

*Correspondence: flexybams@gmail.com
}

How to cite this paper: Barrow, A., Ayobami, A., Azubuike, P. C., \& Cham, D. (2022). Prevalence and Determinants of Acute Respiratory Infections among Children under Five Years in Rural Settings of The Gambia: Evidence from a National Survey. Global Journal of Epidemiology and Infectious Disease, 2(1), 23-32. Retrieved from https://www.scipublications.com/journal/index.php/gjeid/article/view/247

Received: January 19, 2022 Accepted: February 24, 2022 Published: February 25, 2022

Copyright: (c) 2022 by the authors Submitted for possible open access publication under the terms and conditions of the Creative Commons Attribution (CC BY) license (http://creativecommons.org/licenses /by/4.0/).

\begin{abstract}
Background: Acute respiratory infections (ARIs) generally describe a group of infections caused by a range of organisms whose site of action includes the nostrils, through the pharynx to the alveoli. ARIs are reportedly key contributing factors to childhood morbidities and deaths, with a higher impact on children less than the age of five years. This paper aimed at exploring the prevalence of ARIs and their contextual determinants among children less than 60 months of age in the rural settings of the Gambia. Method: The Gambia Demographic and Health Survey (GDHS) in 2019-20 was used for this study. Data were obtained from 1364 rural women aged 15-49 with children less than 60 months through a stratified two-stage cluster sampling approach. Percentages and chi-square tests were used and variables with $p$-value $<0.15$ were included in the model. A multivariable logistic regression model was used to assess the predictors of acute respiratory infection at 95\% confidence interval (CIs) with computed adjusted odds ratios (aORs). All the study data were analyzed using Stata version 17. Results: The weighted prevalence of ARIs among children under 60 months of age in the rural Gambia was 37.1\% with 95\% CI (34.5\% - 39.6\%). The magnitude of ARI was higher among children 25-60 months age group (38.6\%), male children (38.9\%) unvaccinated children $(42.9 \%)$, and those whose fathers and mothers were not working at $60.5 \%$ and $38.7 \%$, respectively. In the adjusted model, children whose mother had primary education $[\mathrm{aOR}=0.65,95 \% \mathrm{CI}=0.46-0.91]$, currently non-breastfed children $[\mathrm{aOR}=1.40,95 \% \mathrm{CI}=1.09-1.79]$ and those whose father were not working [aOR=2.65, 95\% CI=1.47-4.17] were found to be associated with ARIs among children less than 60 months in The Gambia. Conclusion: The prevalence of ARI was moderately high across children under 5 years of age in rural Gambia, low mother's educational levels, and unemployed fathers. The program must consider improvements in the quality of care provided to children in both primary, secondary and tertiary healthcare levels in rural settings. Partner support and adapting community-based supporting systems on child health strategies should be strengthened especially in rural settings.
\end{abstract}

Keywords: Children under-5 years, Acute Respiratory Infections, Prevalence, determinants, The Gambia

\section{Background}

Acute respiratory infections (ARIs) generally describe a group of infections caused by a range of organisms whose site of action include the nostrils, through the pharynx to 
the alveoli $[1,2,3]$. Globally, ARIs are reportedly key contributing factors to childhood morbidities and deaths, with a higher impact on children less than the age of five years [4]. In low-income countries (LICs), there is evidence that each year, an estimated twelve million under-five children are admitted to hospitals owing to acute respiratory infections [5, 6], and 1.3 million deaths associated with ARIs are recorded in this age group [7]. In these low-resource nations, children under the age of five years suffer an average of five ARI episodes annually [8]. In sub-Saharan Africa, ARIs constitute about $6 \%$ of the overall childhood disease burden, which is higher in comparison with that of cancer, diarrhoeal infections, malaria, and HIV $[5,9,10]$. Acute respiratory infections caused about 238 million fatalities in 2016, making them the sixth largest cause of mortality in children under the age of five worldwide [11].

The two major classifications of ARI are the upper respiratory infection (URI) and the lower respiratory infections (LRI) [12], with pneumonia being the commonest LRI and responsible for most morbidities in children [5]. ARIs are caused by viruses (Rhino, Adeno, Corona, Influenza) and bacteria (Klebsiella pneumonia, Haemophilus infuenzae, Streptococcus pyogenes, Staphylococcus aureus) which infect the upper and lower respiratory tracts, including the nose, vocal cords, bronchioles, alveoli, and trachea $[2,6,12]$. In most cases, these conditions may lead to further complications on vital parts of the body, including the membranes around the brain and the ear, and may increase the fatalities of ARI in children [13]. Research has shown that some conditions in children such as infections of the ear, sore throat, difficulties in breathing, and extreme cases as deafness, are traceable to episodes of ARIs that were not treated properly $[2,6,13]$.

Several risk factors of ARIs have been identified in research and these factors vary across low and middle-income nations (LMICs), where there are differences in standards of living, socio-demographic influences, and differential environmental conditions [2, 13]. In these nations, for instance, the child's age, the status of immunization, and biological sex have been linked with ARIs [2,14]. Factors associated with the parents such as the parents' age, employment status, income levels, smoking, and level of education are associated with ARI in children [15, 16]. Such conditions as poverty, poor ventilation, inadequate basic healthcare coverage, urbanization, poor hygiene, and sanitation facilities, and abuse of antibiotics have also been associated with increased risk of ARIs among children $[1,2,17,18]$.

In the Gambia, ARIs are responsible for most morbidities and deaths during childhood, with pneumonia contributing a significant proportion to the nation's burden of childhood illnesses [8]. The Gambian Demographic and Health Survey in 2013 and 2019/20 reported a $28.4 \%$ and $5 \%$ national prevalence of ARI symptoms among children under the age of five years, two weeks before the survey, with only about $70 \%$ of these children given appropriate treatment for the symptoms $[19,20]$. Cost-effective measures exist that help to check ARIs among children. These measures include proper washing of hands, adequate breastfeeding, childhood immunization, and access to attainable methods of timely diagnosis and these measures are strategic to preventing the onset of ARIs $[5,7]$. Despite the prevalence and health implications of ARIs in the Gambia, very few studies have sought to explore the determinants of ARIs among under-five children especially rural settings in the country. This analysis, therefore, seeks to contribute evidence to the determinants of ARIs among rural under-five children in The Gambia, using 2019/2020 GDHS, which is a nationally representative data source.

\section{Methods}

\subsection{Data source}

Data from the 2019-20 Gambia Demographic and Health Survey (GDHS) were used for the analysis, a stratified two-stage cluster sampling approach was used to create a population-based sample. In accordance with the probability proportional to the size of 
the EAs, 281 clusters/Enumerated Areas (EAs) were selected in the first stage of both surveys. The second stage involved methodical selection of 25 households from each cluster/EA. This study randomly extracted a selected rural sample of 1364 women that were interviewed about their children less than 60 months were sampled for those with coughing within the two weeks preceding the study. The study data were derived from 2019-20 rounds of DHS in Gambia that provided information on basic demographic and health indicator including childhood diseases like acute respiratory infections. In Gambia, through the USAID-funded MEASURE DHS programme, ICF International provided technical and financial assistance to the Ministry of Health in collaboration with the Gambia Bureau of Statistics (GBoS) who implemented the survey

\subsection{Variable selection and measurement}

Outcome variables. The study outcome variable was acute respiratory infection outcome among under 5 children at the time of the study. This variable was derived from the question that asked whether a child "had cough in the last two weeks", with a follow up question which asked whether the child "had short, rapid breaths". For the study purpose, acute respiratory infection outcome was recoded into "Yes $=1$ " for those who had cough followed by short, rapid breath, "No $=$ " 0 " for those who had cough but no short nor rapid breath.

Explanatory variables. Twenty-one independent variables categorized into child, parental and household factors were utilized in the study based on a thorough literature review and datasets availability; the variables are listed in Table 1.

Table 1. Definition of independent variables used in the analysis

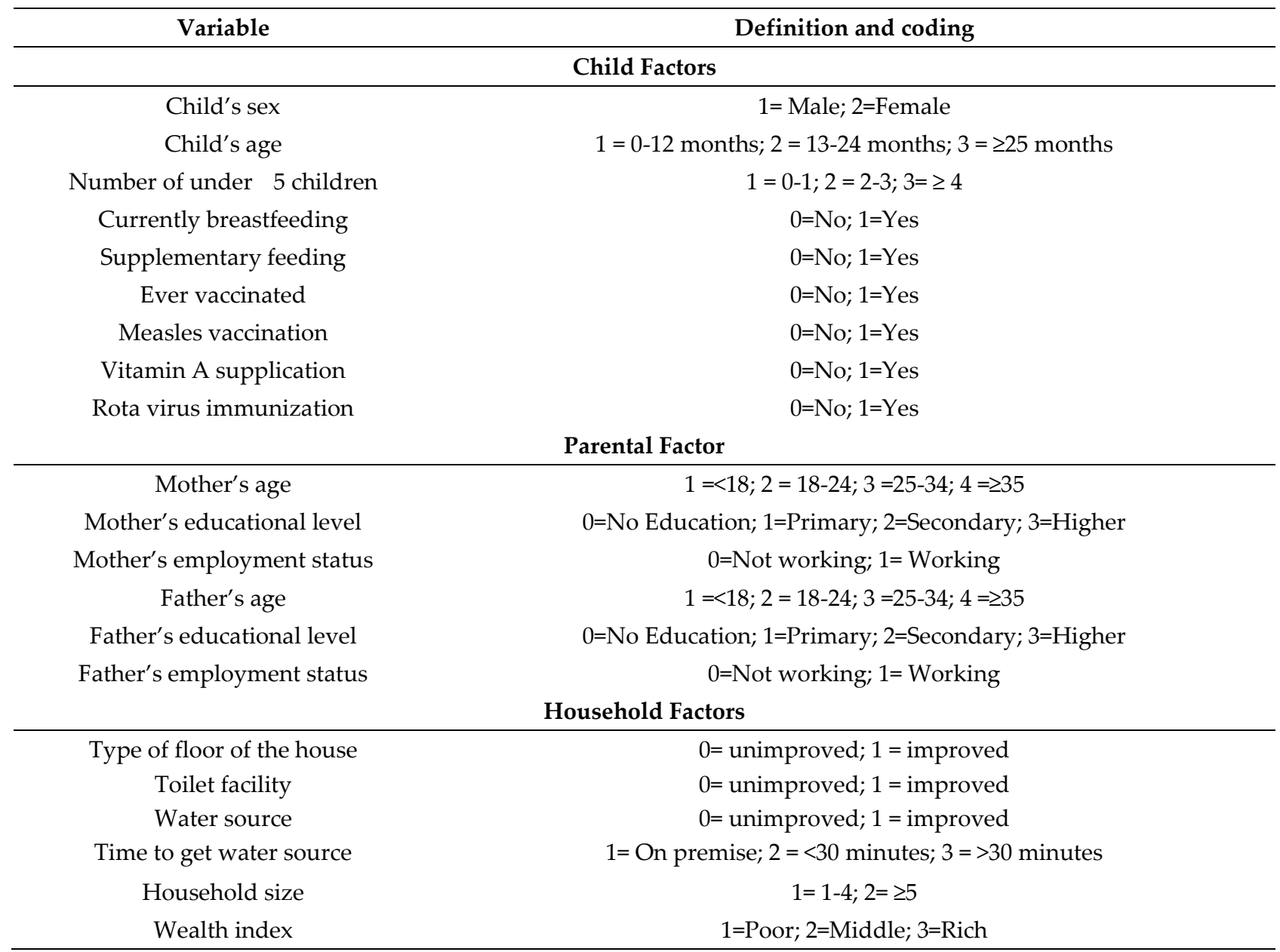




\subsection{Statistical analysis}

The authors conducted descriptive and multivariate analysis. First we used sample weighting to adjust for disproportionate sampling and non-response. For the descriptive analysis, the Pearson's chi-square test was used to identify the association between the outcome variable and the independent variables in their categories. Explanatory variables with a p-value $<0.15$ at bivariate analysis level were considered for inclusion into the multivariable logistic model. Furthermore, a multicollinearity test was conducted for selected variables using cut-off 0.7 [21] and no collinearity was reported across independent variables prior to their inclusion in the model. The final model was adjusted for child sex, currently breadfeeding children, household size, mother's education and father's employment status. We used a multivariable logistic model to adjust for covariables/control variables by including all those that meet our inclusion criteria with computed adjusted odds ratios (aORs) at 95\% confidence interval (CIs). All the study data were analyzed using Stata version 17 .

\subsection{Ethical approval}

The datasets used in this research were population-based datasets that are freely available in the public domain. For reasons of confidentiality, specific characteristics that could be used to identify participants in the study were excluded. As a secondary study, MEASURE DHS/ICF International granted the authors permission to use the datasets. Also, prior to the survey, the DHS project gained ethical approval from the Gambia's Research Ethics Committee.

\section{Results}

\subsection{Prevalence of acute respiratory infection and distribution characteristics of study participants}

Table 2 presents participants characteristics and bivariate analysis of the association between the explanatory variables and ARIs among rural children under-five in the Gambia. ARI proportion among rural children under 5 years in the Gambia was 37.1\% (505 children) with a $95 \% \mathrm{CI}$ of $34.5 \%-39.6 \%$. The proportion of ARI was higher among children within $25-60$ months age group (38.6\%), followed by those within $0-12$ months age group (36.6\%). Higher proportions of male children (38.9\%) reportedly had ARI as compared with female children (34.9\%). Also, the proportion of ARI was higher among children who were not vaccinated $(42.9 \%)$, and whose mother had more than four under-five children (40.1\%). Also, higher proportion of ARI was recorded among children whose father (60.5\%) and mother $(38.7 \%)$ were not working. Similarly, higher ARI proportion was recorded among children whose father $(40.6 \%)$ and mother $(41.0 \%)$ had no education. Also, higher ARI proportion was recorded among children who had unimproved water source (44.4\%) and those who took more than 30 minutes to get water (41.9\%). Bivariate analysis indicated that mother's education and father employment status were significant risk factors associated with ARI among children under-five in rural Gambia. 
Table 2. Bivariate Analysis on Factors Associated to Acute Respiratory Infections among children under 5 years in rural area (GDHS 2019/20)

\begin{tabular}{|c|c|c|c|c|}
\hline \multirow{4}{*}{ Variables } & \multicolumn{3}{|c|}{ ARI } & \multirow{4}{*}{$P$ value } \\
\hline & \multirow{3}{*}{$\begin{array}{c}\text { Rural children } \\
\mathrm{N}=1364\end{array}$} & \multirow{2}{*}{\multicolumn{2}{|c|}{$\begin{array}{c}\text { Rural children under } 5 \\
\text { with ARI }\end{array}$}} & \\
\hline & & & & \\
\hline & & $\mathbf{n}$ & $\%$ & \\
\hline Child's sex & & & & 0.130 \\
\hline Male & 728 & 283 & 38.9 & \\
\hline Female & 636 & 222 & 34.9 & \\
\hline Child's age (in months) & & & & 0.425 \\
\hline $0-12$ & 380 & 139 & 36.6 & \\
\hline $13-24$ & 220 & 110 & 34.4 & \\
\hline$\geq 25$ & 663 & 256 & 38.6 & \\
\hline Number of under five children & & & & 0.274 \\
\hline $0-1$ & 288 & 101 & 35.1 & \\
\hline $2-3$ & 632 & 227 & 35.9 & \\
\hline$>4$ & 444 & 178 & 40.1 & \\
\hline Currently breastfeeding & & & & 0.134 \\
\hline Yes & 807 & 286 & 35.4 & \\
\hline No & 558 & 220 & 39.4 & \\
\hline Supplementary feeding & & & & 0.968 \\
\hline Yes & 304 & 113 & 37.2 & \\
\hline No & 791 & 293 & 37.0 & \\
\hline Ever vaccinated & & & & 0.531 \\
\hline Yes & 61 & 19 & 31.1 & \\
\hline No & 7 & 3 & 42.9 & \\
\hline Measles vaccination & & & & 0.319 \\
\hline Yes & 278 & 109 & 39.2 & \\
\hline No & 657 & 235 & 35.8 & \\
\hline Vitamin A supplication & & & & 0.898 \\
\hline Yes & 720 & 265 & 36.8 & \\
\hline No & 212 & 77 & 36.3 & \\
\hline Rota virus immunization & & & & 0.267 \\
\hline Yes & 803 & 302 & 37.6 & \\
\hline No & 132 & 43 & 32.6 & \\
\hline Mother's age & & & & 0.160 \\
\hline$<24$ & 177 & 59 & 33.3 & \\
\hline $25-34$ & 379 & 147 & 38.8 & \\
\hline$\geq 35$ & 141 & 43 & 30.5 & \\
\hline Mother's education & & & & $0.027^{*}$ \\
\hline No education & 547 & 224 & 41.0 & \\
\hline Primary & 256 & 77 & 30.1 & \\
\hline Secondary & 485 & 175 & 36.1 & \\
\hline Higher & 77 & 30 & 39.0 & \\
\hline Mother's employment status & & & & 0.387 \\
\hline Not working & 421 & 163 & 38.7 & \\
\hline Working & 943 & 342 & 36.3 & \\
\hline Father's age & & & & 0.190 \\
\hline $18-34$ & 311 & 105 & 33.8 & \\
\hline$\geq 35$ & 926 & 351 & 37.9 & \\
\hline Father's education & & & & 0.113 \\
\hline No education & 535 & 217 & 40.6 & \\
\hline
\end{tabular}




$\begin{array}{cccc}\text { Primary } & 84 & 25 & 29.8 \\ \text { Secondary } & 369 & 129 & 35.0 \\ \text { Higher } & 110 & 37 & 33.6\end{array}$

Father's employment status

Not working

$0.001^{*}$

Working

Type of floor of the house

Improved

Unimproved

Toilet facility

Improved

Unimproved

Water source

Stool disposal

$$
\text { Improved }
$$

Unimproved

Time to get water source

On premise

$<30$ minutes

$>30$ minutes

Household size

$\begin{array}{lr}1-4 & 99 \\ \geq 5 & 1264\end{array}$

$\begin{array}{cc}99 & 28 \\ 1264 & 477\end{array}$

41.9

Wealth Index

Poor

498

28.3

37.7

$\begin{array}{cc}\text { Poor } & 498 \\ \text { Middle } & 275 \\ \text { Rich } & 590\end{array}$

* Statistical significance at $\mathrm{p}<0.05$

\subsection{Determinants of ARI among under-five children rural Gambia (GDHS 2019/20)}

Table 3 shows the logistic regression results on the factors associated with ARI among under-five children rural Gambia. In the adjusted model, children whose mother had primary education had significant lower odds $[\mathrm{aOR}=0.65,95 \% \mathrm{CI}=0.46-0.91]$ of having ARI compared to those with no education. Furthermore, children not currently breastfeeding had higher odds [aOR=1.40, 95\% CI=1.09-1.79] of developing ARI compared to those currently breastfeeding. Finally, children whose father were not working had higher odds [aOR=2.75, 95\% CI=1.47-54.17] of developing ARI compared to those whose fathers were working after controlling for confounders. 
Table 3. Determinants of ARI among under-five children rural Gambia (GDHS 2019/20)

\begin{tabular}{cc}
\hline & GDHS 2019/20 data \\
\cline { 2 - 2 } Variables & ARI \\
\cline { 2 - 2 } Child's sex & Yes vs No \\
Male & 1 \\
Female & $0.89(0.69-1.120$ \\
Yes & 1 \\
No & $1.40(1.09-1.79)^{*}$ \\
Currently breastfeeding & 1 \\
1-4 & $1.49(0.93-2.41)$ \\
$\geq 5$ & 1 \\
Household size & $0.65(0.46-0.91)^{*}$ \\
No education & $0.96(0.72-1.27)$ \\
Primary & $1.04(0.57-1.87)$ \\
Secondary & \\
Higher & 1 \\
Working & $2.75(1.47-5.17)^{*}$ \\
Not working &
\end{tabular}

aOR: Adjusted Odds Ratio, * Statistical significance at $\mathrm{p}<0.05$

\section{Discussion}

The study aimed at determining the factors associated with Acute Respiratory Infections among under-five children using data from The Gambia 2019/2020 DHS. The ARI proportion among children under 5 years in the Gambia, analyzed to be $37.1 \%$ may have been because the data collection was done during the dry season (November 2019 to March 2020), which mainly has the features of dusty cold and dry winds due to the harmattan. This prevalence is slightly higher than the initially reported $5 \%$ national prevalence since the current study computed ARI prevalence only from those under five children from rural areas in the last two weeks preceding the survey. This proportion, although high, is relatively lower than ARI proportions obtained from studies in Cameroon, 54.7\% [18], Kenya, 69.7\% [16], and higher than the ARI prevalence obtained in Ethiopia, $26.3 \%$ [4]. This finding was expected as rural areas are typically prone to dust and dry airs, compared with the urban residence where the presence of industries and urbanization may limit exposure to dusty regions. Dry seasons have been reported to have a higher effect in rural than in urban regions, concerning creating a risk of ARI symptoms, especially among preschool children [1, 2]. Our finding is comparable to studies that have found an independent and significant association between ARI prevalence based on geographical locations of survey samples [4, 8, 22, 23]

Similarly, the study recorded a higher ARI proportion among children whose parents had no form of formal education. Furthermore, children of mothers with primary education had significantly lower odds $[\mathrm{aOR}=0.64,95 \% \mathrm{CI}=0.46-0.90]$ of having ARI compared to those whose mothers had no formal education. This finding was not surprising as education empowers individuals to make informed health choices for themselves and people around them, with literacy level affecting the awareness of and access to health information by caregivers. Hence, being an educated mother may have informed her choice of taking up postnatal healthcare services such as immunization which mitigates ARI among children. Evidence exists that among the social determinants of health, education has been a predominant construct as various studies, especially in the developing countries have 
noted the educational status of mothers and caregivers as an important predictor of under-five health status and overall family health outcomes [18, 24]. This finding is also comparable with results from the Kenya 2014 DHS where incomplete primary education or no formal education of caregivers resulted in their infants having a 1.49 times higher risk of ARI when compared to children of educated mothers [16].

In addition, the occupational status of fathers showed a significant association with the proportion of ARI recorded among under-five children in the study. Analysis showed that children whose fathers were not working had higher odd $[\mathrm{aOR}=2.65,95 \% \mathrm{CI}=1.42$ 4.95] of developing ARI compared to those whose fathers were working. Fathers' occupational status, especially in developing countries like the Gambia is an indicator of the wealth status of the family, as fathers are mostly breadwinners in Gambian families especially in rural settings. Hence, the wealth quintile of households have been implicated as an explanatory variable to the differential prevalence of ARI and other childhood morbidities [16], with children from families that are wealthier reported in studies to be less likely to suffer from ARI than children from households categorized as poor [12, 14]. The explanation for this may be that wealthier families ensure better healthcare and nourishment for their children. In addition, studies have associated poverty with such risk factors of ARI as overcrowding, low uptake of healthcare services, use of unclean cooking fuel, and malnutrition $[7,16,18]$.

\section{Strength and limitation}

We used a nationally representative dataset, ensuring that the study's results can be generalized to Gambia's children under five years of age in rural settings. In addition, due to the large sample size, extensive reporting of ARI prevalence was possible. However, the studies used cross-sectional data, implying that no causal relationships were determined.

\section{Conclusion}

The prevalence of ARI was moderately high across children under 5 years of age in rural Gambia, low mother's educational level, and unemployed fathers. The program must consider improvements in the quality of care provided to children in both primary, secondary and tertiary healthcare levels in rural settings. Partner support and adapting community-based supporting systems on child health strategies should be strengthen especially in rural settings. Future studies should look into the healthcare systems and service-related factors that could hinder the quality and children's utilization of services across rural settings.

\section{Declarations}

\section{Acknowledgement}

The authors thank the MEASURE DHS project for their support and for free access to the original data.

\section{Funding}

The authors have no support or funding to report.

\section{Availability of data and materials}

Data for this study were sourced from Demographic and Health surveys (DHS) and available here: https://www.dhsprogram.com/data/available-datasets.cfm 
$\mathrm{AB}, \mathrm{AA}, \mathrm{AP} \& \mathrm{DC}$ conceptualized the study and prepared the study design, reviewed literature, analysis of data and wrote the results. All authors critically reviewed the manuscript for its intellectual content. $\mathrm{AB}$ had final responsibility to submit for publication.

\section{Competing interests}

The authors declare that they have no competing interests.

\section{Ethics approval and consent to participate}

Ethics approval for this study was not required since the data is secondary and is available in the public domain. More details regarding DHS data and ethical standards are available at: http://goo.gl/ny8T6X.

\section{Consent for publication}

No consent to publish was needed for this study as we did not use any details, images or videos related to individual participants. In addition, data used is available in the public domain.

\section{References}

[1] Adesanya OA, Chiao C. Environmental risks associated with symptoms of acute respiratory infection among preschool children in north-western and south-southern Nigeria communities. Int J Environ Res Public Health. 2017;14.

[2] Akinyemi JO, Morakinyo OM. Household environment and symptoms of childhood acute respiratory tract infections in Nigeria, 2003-2013: A decade of progress and stagnation. BMC Infect Dis. 2018;18:1-12.

[3] Greenwood B. Epidemiology of Acute Lower Respiratory Tract Infections, Especially Those Due to Haemophilus influenzae Type b, in The Gambia, West Africa. J Infect Dis. 1992;165 Supplement 1:S26-8.

[4] Alemayehu M, Alemu K, Sharma HR, Gizaw Z, Shibru A. Household Fuel Use and Acute Respiratory Infections in Children Under Five Years of Age in Gondar city of Ethiopia. J Environ Earth Sci. 2014;4:77-86.

[5] Seidu AA, Dickson KS, Ahinkorah BO, Amu H, Darteh EKM, Kumi-Kyereme A. Prevalence and determinants of Acute Lower Respiratory Infections among children under-five years in sub-Saharan Africa: Evidence from demographic and health surveys. SSM - Popul Health. 2019;8 February:100443.

[6] Asghar SA, Srivastava MR, Srivastava JP, Gupta P, Zaidi ZH. Prevalence of acute respiratory infections among children under five years of age attending rural health training centre of Era's Lucknow Medical College and Hospital. Int J Community Med Public Health. 2017;4:3752.

[7] Kumar GS, Majumdar A, Kumar V, Naik BN, Selvaraj K, Balajee K. Prevalence of acute respiratory infection among under-five children in urban and rural areas of puducherry, India. J Nat Sci Biol Med. 2015;6:3-6.

[8] Mackenzie GA, Hill PC, Sahito SM, Jeffries DJ, Hossain I, Bottomley C, et al. Impact of the introduction of pneumococcal conjugate vaccination on pneumonia in The Gambia: population-based surveillance and case-control studies. Lancet Infect Dis. 2017;17:965-73.

[9] Simen-Kapeu A, Bogler L, Weber A-C, Ntambi J, Zagre NM, Vollmer S, et al. Prevalence of diarrhoea, acute respiratory infections, and malaria over time (1995-2017): A regional analysis of 23 countries in West and Central Africa. J Glob Health. 2021;11:13008.

[10] Wardlaw T, Salama P, Johansson EW, Mason E. Pneumonia: the leading killer of children. The Lancet. 2006;368:1048-50.

[11] Troeger C, Blacker B, Khalil IA, Rao PC, Cao J, Zimsen SRM, et al. Estimates of the global, regional, and national morbidity, mortality, and aetiologies of lower respiratory infections in 195 countries, 1990-2016: a systematic analysis for the Global Burden of Disease Study 2016. Lancet Infect Dis. 2018;18:1191-210.

[12] BROWNLEY HC. Acute respiratory infections. Va Med Mon. 1949;76:63-8.

[13] Banda W, Mazaba ML, Mulenga D, Siziya S. Risk factors associated with acute respiratory infections among under-five children admitted to Arthur's Children Hospital, Ndola, Zambia. Asian Pac J Health Sci. 2016;3:153-9.

[14] Nair H, Simões EAF, Rudan I, Gessner BD, Azziz-Baumgartner E, Zhang JSF, et al. Global and regional burden of hospital admissions for severe acute lower respiratory infections in young children in 2010: A systematic analysis. The Lancet. 2013;381:1380-90.

[15] Cardoso AM, Coimbra CEA, Werneck GL. Risk factors for hospital admission due to acute lower respiratory tract infection in Guarani indigenous children in southern Brazil: A population-based case-control study. Trop Med Int Health. 2013;18:596-607. 
[16] Mulatya DM, Mutuku FW. Assessing Comorbidity of Diarrhea and Acute Respiratory Infections in Children Under 5 Years: Evidence From Kenya's Demographic Health Survey 2014. J Prim Care Community Health. 2020;11.

[17] Tekle AG, Worku A, Birhane Y. Factors associated with acute respiratory infection in children under the age of 5 years: evidence from the 2011 Ethiopia Demographic and Health Survey [Corrigendum]. Pediatr Health Med Ther. $2015 ;: 129$.

[18] Tazinya AA, Halle-Ekane GE, Mbuagbaw LT, Abanda M, Atashili J, Obama MT. Risk factors for acute respiratory infections in children under five years attending the Bamenda Regional Hospital in Cameroon. BMC Pulm Med. 2018;18:1-8.

[19] The Gambia Bureau of Statistics - GBOS, ICF International. The Gambia Demographic and Health Survey 2013. Banjul, The Gambia: GBOS and ICF International; 2014.

[20] Gambia Bureau of Statistics - GBoS, ICF. The Gambia Demographic and Health Survey 2019-20. Banjul, The Gambia: GBoS/ICF; 2021.

[21] Midi H, Sarkar SK, Rana S. Collinearity diagnostics of binary logistic regression model. J Interdiscip Math. 2010;13:253-67.

[22] Fera D, Sriwahyuni S. The Relationship between Home Environmental Conditions and the Occurrence of Acute Respiratory Infection (ARI) in Toddlers in Nagan Raya Regency. J-Kesmas J Fak Kesehat Masy Indones J Public Health. 2020 ;7:38.

[23] Costilla-Esquivel A, Corona-Villavicencio F, Velasco-Castañón JG, Medina-De La Garza CE, Martínez-Villarreal RT, CortesHernández DE, et al. A relationship between acute respiratory illnesses and weather. Epidemiol Infect. 2014;142:1375-83.

[24] Zimmerman E, Woolf SH. Understanding the Relationship Between Education and Health. NAM Perspect. 2014;4. 